\title{
Development of an acute pancreatitis porcine model based on endoscopic retrograde infusion of contrast medium or sodium taurocholate
}

\author{
Jin-Seok Park ${ }^{1}$, Seok Jeong ${ }^{1,2}$, Joon Mee Kim³ ${ }^{3}$ Bum Hei Lee ${ }^{2}$, Jae Min Kim², and Don Haeng Lee ${ }^{1,2}$
}

\begin{abstract}
${ }^{1}$ Digestive Disease Center, Department of Internal Medicine, Inha University School of Medicine, Incheon; ${ }^{2}$ The National Center of Efficacy Evaluation for the Development of Health Products Targeting Digestive Disorders (NCEED), Incheon; ${ }^{3}$ Department of Pathology, Inha University School of Medicine, Incheon, Korea
\end{abstract}

Received: November 2, 2017

Revised : May 9, 2018

Accepted: May 22, 2018

\section{Correspondence to}

Seok Jeong, M.D.

Department of Internal Medicine, Inha University Hospital, 27 Inhang-ro, Jung-gu, Incheon 22332, Korea

Tel: $+82-32-890-2548$

Fax: +82-32-890-2549

E-mail:inos@inha.ac.kr

\section{Don Haeng Lee, M.D.}

Department of Internal

Medicine, Inha University

Hospital, 27 Inhang-ro, Jung-gu,

Incheon 22332, Korea

Tel: $+82-32-890-2548$

Fax: +82-32-890-2549

E-mail: LDH@inha.ac.kr
Background/Aims: A reproducible, endoscope-based, large animal model, of acute pancreatitis was developed to meet the need for a suitable means of preclinically testing treatments. The aim of this study was to develop an endoscope-based animal model of acute pancreatitis.

Methods: This experimental study was conducted on six mini-pigs. The pancreatitis model was induced by infusing contrast medium (CM) or sodium taurocholate (TCA) under high pressure $(100 \mathrm{mmHg})$ into the main pancreatic duct by endoscopic retrograde pancreatography. Animals were randomly allocated to three groups: a CM group, a 10\% TCA group, and a 20\% TCA group. Pancreatic injuries were evaluated histologically, and serum amylase and lipase levels were measured.

Results: Acute pancreatitis was observed in all animals during hematologic and histologic examinations. Serum amylase and lipase levels were significantly higher (> 10 times baseline), and pancreatic edema, vacuolization of acinar cells, and hemorrhagic necrosis were observed. Severity of pancreatitis tended to be greater in the TCA groups than in the CM group as assessed using histologic scores, and degrees of pancreatitis were found to be dose-dependently related to TCA concentration.

Conclusions: The two endoscopic procedures described are effective and safe for creating a swine model of acute pancreatitis. The authors hope the described endoscopic methods will assist in the development of a suitable treatment strategy.

Keywords: Pancreatitis; Animals; Endoscopes

\section{INTRODUCTION}

Acute pancreatitis is an inflammatory disorder that affects the pancreas and surrounding organs. In about
$85 \%$ to $80 \%$ of cases, acute pancreatitis follows a benign clinical course, which responds well to conservative treatment, but remainder develop severe hemorrhagic necrotizing pancreatitis, which has a grave clinical prog- 
nosis and is often accompanied by multi-organ failure despite intensive treatment or surgery [1]. Unfortunately, effective treatment strategies are limited due to poor understanding of the pathogenesis of this disease [2], and thus, studies are required to investigate the mechanism of acute pancreatitis. However, studies during different disease stages are limited by the anatomical location of the organ [3], and thus, an animal model of acute pancreatitis is needed to facilitate such studies.

Many investigators have sought to establish a satisfactory model of experimental pancreatitis based on the infusion of a noxious agent in a retrograde manner into the pancreatic duct or by pancreatic duct ligation [4-6]. However, previously devised models of pancreatitis have usually required laparotomy, which requires complicated preparation and postoperative care, such as, analgesics, antibiotics, and wound care. Furthermore, surgery introduces the risks of procedure related-infections, and thus, hinders estimations of inflammatory reactions attributable to pancreatitis. Therefore, we undertook to develop a large animal model of acute pancreatitis based on the delivery of a noxious agent by endoscopic retrograde infusion into the pancreatic duct. In particular, we examined the abilities of contrast medium (CM) and sodium taurocholate (TCA) to develop acute pancreatitis in a large animal model and compared the severities of acute pancreatitis caused by CM and TCA infusions.

\section{METHODS}

\section{Animals}

Six mini pigs (Sus scrofa, mean age 14 months, mean body weight $30 \mathrm{~kg}$ ) were used in the study. Two animals were randomly allocated to each of three groups, namely, the CM group, the 10\% TCA group, and the 20\% TCA group. Animals were fasted for 24 hours before endoscopic procedures but allowed water. Pre-anesthesia sedation was conducted by injecting atropine sulfate ( 0.04 $\mathrm{mg} / \mathrm{kg}$ ), xylazine (2 mg/kg), and tiletamine-zolazepam (5 $\mathrm{mg} / \mathrm{kg}$ ) intramuscularly. After intubation, general anesthesia was achieved using $0.5 \%$ to $2 \%$ isoflurane through an endotracheal tube in $70 \%$ nitrous oxide/30\% oxygen provided by a ventilator. Animals were then placed on their sides on a fluoroscopy table. Cardiopulmonary pa- rameters were monitored throughout procedures. The study protocol was approved by our Institutional Animal Care Committee before study commencement (IACUC 17-KE-389).

\section{Retrograde infusion of CM or TCA through the main pancreatic duct}

Endoscopic retrograde pancreatography (ERP) was performed using a side view duodenoscope (TJF-24O, Olympus Co. Ltd., Tokyo, Japan). After inserted into the duodenum, the endoscope was positioned close to the orifice of the pancreatic duct, which is usually located in the and portion of duodenum. Pancreatic duct cannulation (endoscopic retrograde cholangiopancreatography [ERCP] catheter, bottle-shaped metal tip, MTW-Endoskopie, Wesel, Germany) was performed by wire-guided cannulation using a 0.035 -inch hydrophilic tipped guidewire (Boston Scientific Corp., Natick, MA, USA). Because the pancreatic duct orifice of mini pigs does not have a sphincter, sphincterotomy is not required for cannula insertion or for other endoscopic procedures. Pancreatography was achieved through the cannula. CM was injected to confirm pancreatic duct cannulation until the pancreatic duct was partially visualized by fluoroscopy. The cannula was then gently inserted to the pancreas tail to prevent regurgitation of noxious agent. CM (10 mL, low-osmolarity iodinated contrast, Omnipaque, Amersham Health, Princeton, NJ, USA) or TCA (10 mL; $10 \%$ or $20 \%$ ) was infused through the cannula at a pressure of $100 \mathrm{mmHg}$ until acinarization was observed by fluoroscopy (Fig. 1). The infusion pressure was controlled manually using inflation device (Indeflator, Abbott, Santa Clara, CA, USA) by one experienced assistant.

\section{Biochemical measurements}

Serum amylase and lipase levels were determined by blood sampling from a jugular vein prior to the procedure, and at 1, 2, 18, and 24 hours after the procedure.

\section{Necropsy and pathology assessments}

Animals were sacrificed 24 hours after the procedure by pentobarbital injection (100 mg/kg intravenous) and pancreata were removed by laparotomy. Acute pancreatitis was evaluated macroscopically during necropsy. Histologic examinations of pancreata were performed on all six animals. 


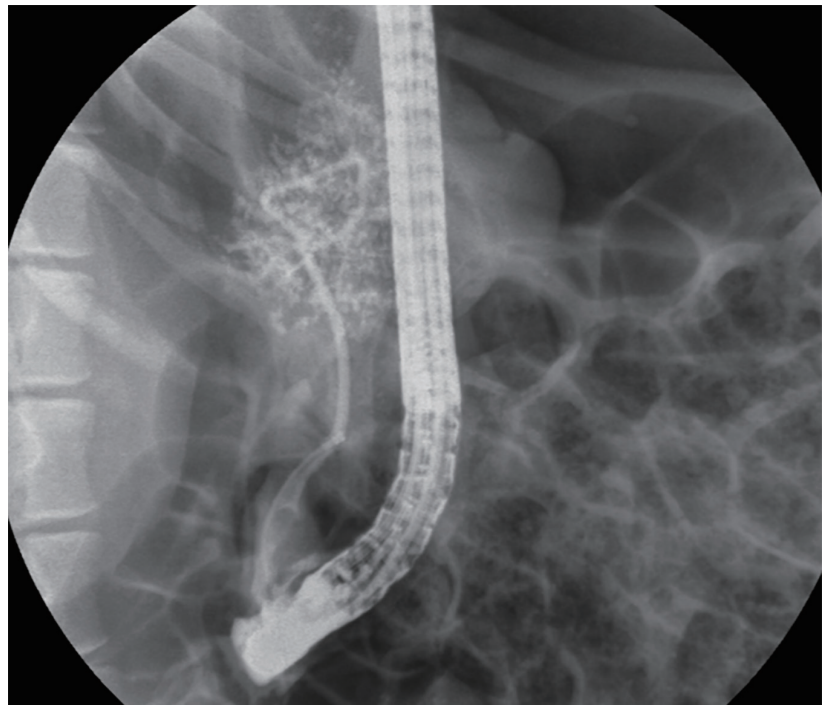

Figure 1. Fluoroscopic images demonstrating acinarization of pancreatic parenchyma by contrast medium infusion.

\section{Histological scoring of acute pancreatitis}

Removed tissues were fixed in neutral buffered formalin and serially sectioned at 5-mm intervals. Multiple cross-sections were taken from pancreatic heads to tails, and stained with hematoxylin and eosin (H\&E) for microscopic examination. These sections were then evaluated by one expert pathologist unaware of specimen identities. Pancreatitis was graded for degree of interstitial edema, leukocyte infiltration, vacuolization, necrosis (acinar cell and fat), and hemorrhage using a 0 to 4 scale, as previously described for the histologic scoring for acute pancreatitis (Table 1) [7-9].

\section{Statistical analysis}

Continuous variables are expressed as mean \pm standard deviation (SD). The quantitative variables were com-

Table 1. Histological scores of acute pancreatitis

\begin{tabular}{|c|c|c|}
\hline Condition & Score & Description \\
\hline \multirow[t]{5}{*}{ Edema } & 0 & Absent \\
\hline & 1 & Diffuse expansion of interlobar septa \\
\hline & 2 & 1+ Diffuse expansion of interlobular septa \\
\hline & 3 & 2+ Diffuse expansion of interacinar septa \\
\hline & 4 & 3+ Diffuse expansion of intercellular septa \\
\hline \multirow[t]{5}{*}{ Inflammation } & o & Absent \\
\hline & 1 & Around ductal margin \\
\hline & 2 & In parenchyma (<50\% of lobules) \\
\hline & 3 & In parenchyma ( $51 \%-75 \%$ of lobules) \\
\hline & 4 & In parenchyma (75\% of lobules) \\
\hline \multirow[t]{5}{*}{ Vacuolization } & o & Absent \\
\hline & 1 & Periductal (5\%) \\
\hline & 2 & Focal $(5 \%-20 \%)$ \\
\hline & 3 & Diffuse $(21 \%-50 \%)$ \\
\hline & 4 & Severe $(50 \%)$ \\
\hline \multirow[t]{5}{*}{ Necrosis } & o & Absent \\
\hline & 1 & $<10 \%$ of the section affected by abnormal lesion \\
\hline & 2 & $10 \%-33 \%$ of the section affected by abnormal lesion \\
\hline & 3 & $33 \%-66 \%$ of the section affected by abnormal lesion \\
\hline & 4 & $>66 \%$ of section affected by abnormal lesion \\
\hline \multirow[t]{5}{*}{ Hemorrhage } & o & Absent \\
\hline & 1 & $<10 \%$ of the section affected by abnormal lesion \\
\hline & 2 & $10 \%-33 \%$ of the section affected by abnormal lesion \\
\hline & 3 & $33 \%-66 \%$ of the section affected by abnormal lesion \\
\hline & 4 & $>66 \%$ of section affected by abnormal lesion \\
\hline
\end{tabular}



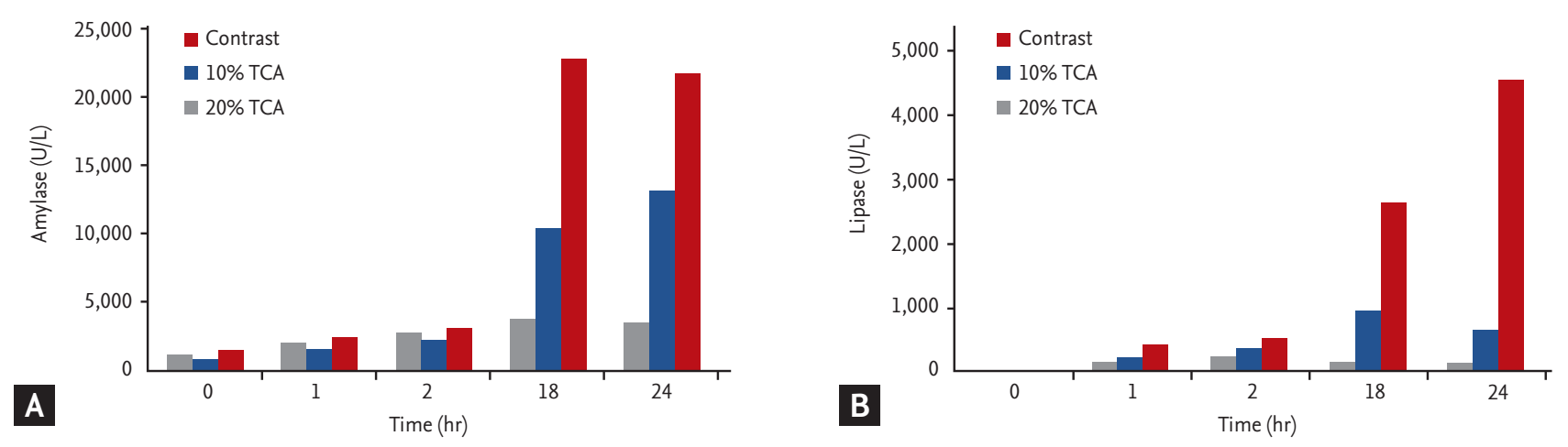

Figure 2. Baseline and mean peak levels of amylase (A) and lipase (B) at 1, 2, 18, and 24 hours after the procedure. TCA, taurocholate.

pared using the Mann-Whitney U test. Differences with $p$ values of $<0.05$ were considered significant. Data were analyzed using SPSS version 19.0 for Windows (IBM Co., Armonk, NY, USA).

\section{RESULTS}

\section{Feasibility and safety}

Retrograde infusions of CM or TCA were successfully performed in all animals, and no technical difficulties were encountered during the procedures. Pancreatic acinarization was accomplished in all cases. No adverse events, such as, hemodynamic instability, localized bleeding, or damage to duodenal lumen were observed. All animals survived the examination period and developed acute pancreatitis within 24 hours of the procedure.

\section{Biochemical determination}

Serum amylase and lipase levels before and 1, 2, 18, and 24 hours after the procedure are presented in Fig. 2. Significant increases in serum amylase and lipase levels were noted in all animals. Serum amylase and lipase levels were at least three times greater at all post-procedural time points than at baseline in all six animals. Furthermore, mean serum amylase levels were significantly higher in the TCA group (the 10\% and 20\% TCA groups combined) than in the CM group at 24 hours after the procedure, as determined by the paired Student's $t$ test $(15,728.4 \pm 10,183.7$ international unit [IU] vs. $3,399.75 \pm 471.99$ IU). Likewise, mean serum lipase was also higher in the TCA $(2,684.5 \pm 27,007$ IU $)$ than in the
CM group (139.3 $\pm 27.2 \mathrm{IU})$.

\section{Histologic examination}

On macroscopic examination, morphological changes, which included focal hemorrhage and necrosis, were observed in the pancreata of all animals (Fig. 3A). In addition, peripancreatic fluid collection was noted in the 10\% and 20\% TCA groups (Fig. 3B). Microscopic examinations showed all six animals developed interstitial edema, vacuolization, necrosis, and hemorrhage. Furthermore, histologic changes were observed in all pancreatic sections beyond the injection area, and degrees of histologic changes in the pancreas head, body, and tail were similar regardless of injection area. However, degrees of acute pancreatitis depended on the injection agent, that is, CM induced mild pancreatitis, whereas TCA caused severe pancreatitis (Fig. 4). Histologic pancreatitis scores for the CM and TCA groups are provided in Table 2. The overall mean histologic total pancreatitis score for the six animals was $10 \pm 1.37$, and mean pancreatitis score in the CM group $(6.5 \pm 0.71)$ was significantly lower than in the TCA group $(12.0 \pm 2.45, p=0.02)$. No relation was observed between the severity of acute pancreatitis and TCA concentration $(p=0.29)$. However, necrosis scores did appear to be related to TCA concentration (mean necrosis scores in the $10 \%$ and $20 \%$ TCA groups were 2.5 and 4 , respectively; $p=0.10$ ).

\section{DISCUSSION}

This pilot study shows that endoscopic retrograde infusion of CM or TCA into the pancreatic duct provides a 


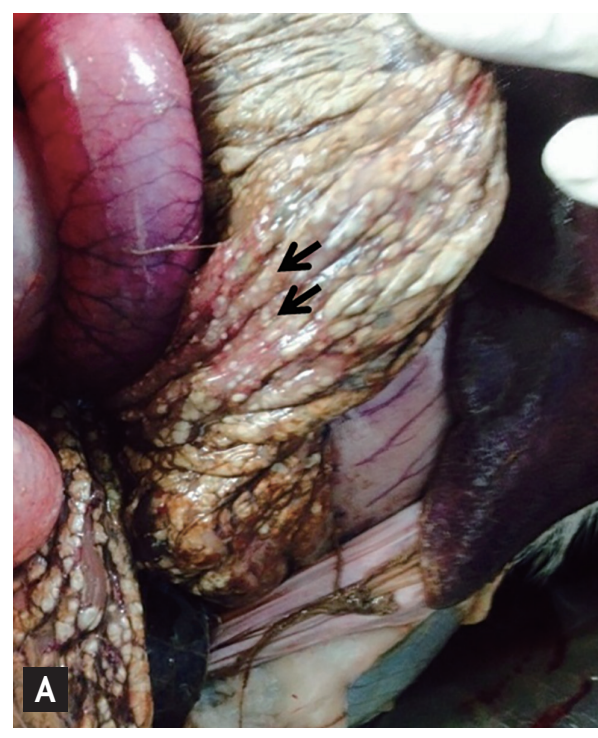

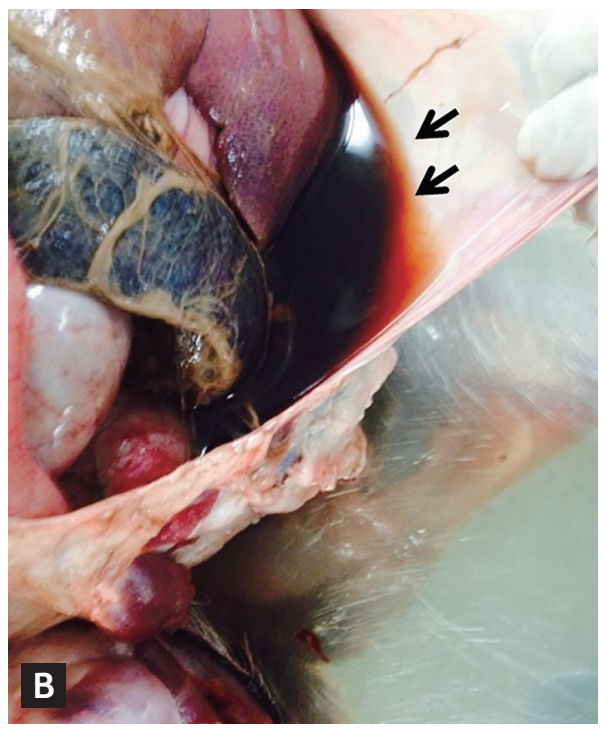

Figure 3. Macroscopic examination of pancreas. (A) Focal hemorrhage and necrosis was detected in all animals (arrows). (B) Peripancreatic fluid collections were noted in the $10 \%$ and $20 \%$ taurocholate groups (arrows).
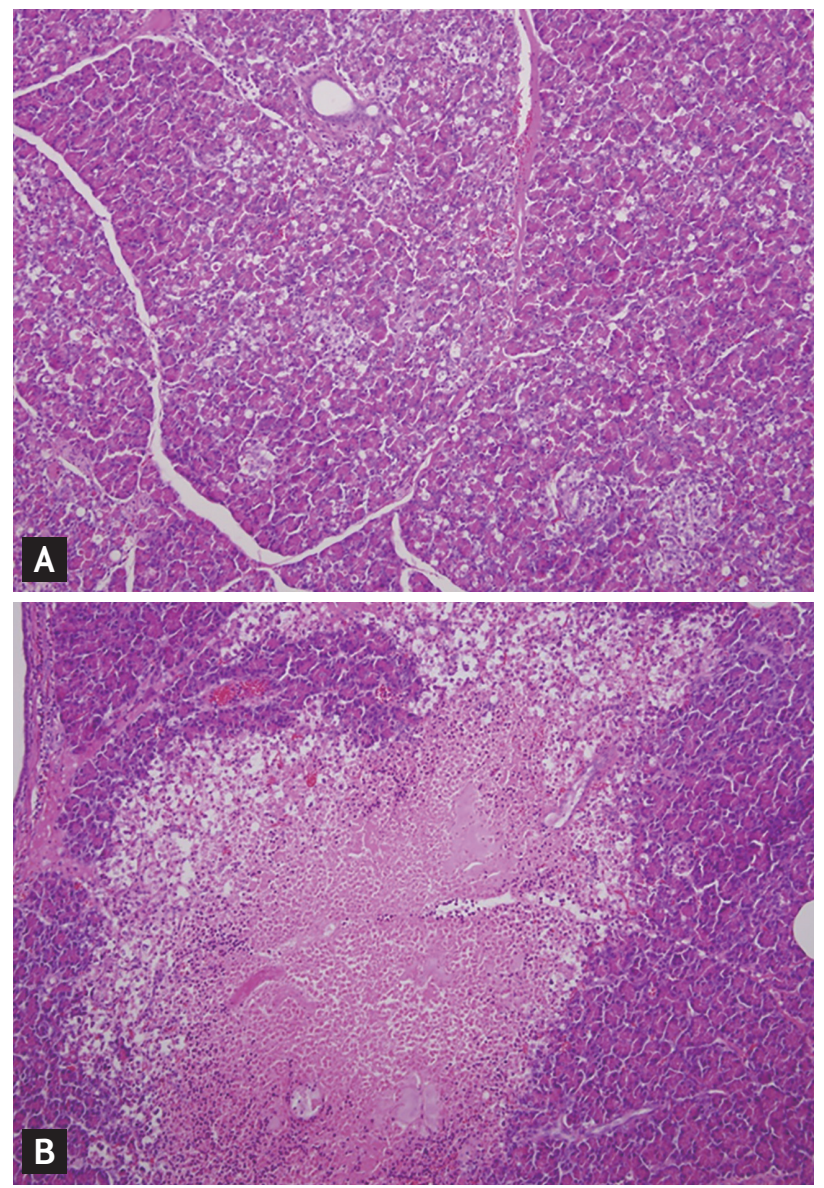

Figure 4. Histologic analysis of pancreatic sections after noxious agent injection. (A) Representative section from the contrast medium group showing mild leukocytic infiltration, minimal interlobar edema, and scattered vacuolization. (B) Representative section from the $20 \%$ taurocholate group showing marked leukocytic infiltration, vacuolization, inter-and intralobular edema, hemorrhage, and extensive necrosis (H\&E, $\times 100)$. safe, effective means of producing a porcine model of acute pancreatitis. In the current study, acute pancreatitis developed in all animals, and the results showed that its degree can be controlled by using different types of noxious agent.

The method used to produce our animal model has some advantages. First, the procedure is straightforward, as in the present study, endoscopic retrograde infusion was performed without technical difficulty within half an hour. However, investigators not used to large animal endoscopic experiments may experience difficulty finding the pancreatic papilla in the duodenum. The porcine pancreatic duct enters the second portion of the duodenum and is separated from and distal to the bile duct opening [10]. The pancreatic papilla appears as a pin-point hole, whereas the bile duct opening can be readily identified as a firm whitish structure. Therefore, careful observation of the duodenal wall after passing the bile duct opening is essential to locate the pancreatic papilla in pigs with an endoscope. Cannulation of the pancreatic duct is performed using a wire-guided method similar to that used to cannulate the common bile duct in swine.

The second advantage of the procedure is that it is less invasive than the surgical procedures used to produce large animal models of acute pancreatitis. To expose the pancreatic duct surgically, a large abdominal incision is made from the xiphoid to the umbilicus $[11,12]$, and thus, surgery introduces the risk of proce- 
Park JS, et al. Acute pancreatitis model based on endoscopic noxious agents infusion

Table 2. Histologic pancreatitis scores in each model

\begin{tabular}{lcccccc}
\hline & Edema & Inflammation & Vacuolization & Necrosis & Hemorrhage & Total score \\
\hline Contrast & 1 & 1 & 3 & 1 & 1 & 7 \\
Contrast & 1 & 0 & 3 & 1 & 1 & 1 \\
$10 \%$ TCA & 2 & 1 & 3 & 3 & 2 & 9 \\
$10 \%$ TCA & 2 & 1 & 4 & 4 & 4 & 12 \\
$20 \%$ TCA & 3 & 0 & 4 & 4 & 1 & 12 \\
\hline $20 \%$ TCA & 2 & 1 & 4 & 2 & 12 \\
\hline
\end{tabular}

TCA, sodium taurocholate.

dure-related adverse events, such as, infections, bleeding, and mortality. In addition, the accurate estimation of pancreatitis severity might not be possible in such models because interactions between surgical trauma and acute pancreatitis are likely to adversely affect assessments. Furthermore, anesthesia and operative procedures often aggravate or even potentiate pancreatitis [13]. On the other hand, endoscopic retrograde pancreatic duct infusion is comparatively free of such complicating factors, and thus, enables accurate assessments of acute pancreatitis severity. It should also be mentioned that no adverse event or mortality was encountered in current study.

The third advantage is that the model allows pancreatitis severity to be controlled. Although it is well known that inflammatory processes play an important role in acute pancreatitis, current knowledge as to how these inflammatory processes result in diverse clinical presentations and sometimes in organ failure are not well understood, because of the anatomical location of the pancreas and difficulties acquiring tissues at different disease stages [14]. The model was devised to address this problem by utilizing the retrograde infusion of noxious agents into the pancreatic duct, as is commonly employed in models of experimental pancreatitis. However, in previously described models the severity of pancreatitis was highly variable and difficult to control. We believe this variability was caused by the administration of different infusion agent volumes or the use of different infusion pressures. In contrast, we accurately controlled pressure and volume at infusion, and found that pancreatitis severity could be controlled by using different infusion agent types or different concentrations.
The last advantage is that our large animal model is a proper animal model could contribute to the preclinical animal research for the development of new endoscopic treatment or devices. Various animal models have been described and the selection of a particular model depends upon the specific aims of the experiment. Small animals including rats and mice were mainly used to evaluate the pathophysiology of pancreatitis. However, these small models cannot be used in the development of endoscopic treatment because of the anatomical difference with human. In contrast with small pancreatitis animal model, the condition of large experimental models is similar to the clinical manifestations seen in human patients with acute pancreatitis and all endoscopic procedures can be performed. Thus, large animal model can provide clinicians to practice endoscopic therapeutic techniques, such as endoscopic necrosectomy as well as to conduct preclinical experiments prior to working with human.

Regarding the severity of pancreatitis, TCA was found to be better than $\mathrm{CM}$ at producing severe pancreatitis, which may have been caused by mechanistic differences. The mechanism responsible for the effect of TCA (a bile salt) has been postulated to be due to its detergent effect, whereas hydrostatic injury caused by pressure at injection has been suggested to underlie CM induced acute pancreatitis $[15,16]$. In addition, the severity of pancreatitis, especially as determined by acinar cell necrosis and fatty tissue necrosis, tended to increase with TCA concentration (necrosis scores of $10 \%$ TCA vs. $20 \%$ TCA were 2.5 and 4, respectively), and in our opinion the lack of a significant difference between these scores was due to small sample numbers. Furthermore, our results concurs with those of Wittel et al. [17], who 
demonstrated that animals administered $4 \%$ or $5 \%$ TCA had significantly more areas of mesenteric and retroperitoneal fatty tissue necrosis than animals administered $2 \%$ TCA.

The acute pancreatitis induced by CM in current model was similar to that observed for post-ERCP pancreatitis. ERCP has been commonly used to treat biliopancreatic disease for about 40 years and post-procedural acute pancreatitis, which has an incidence of between $1 \%$ and $22 \%[18,19]$, continues to be a feared complication. Furthermore, although the risk factors of post-ERCP pancreatitis, which include, pancreatic duct injection, pancreatic sphincterotomy, difficult or failed cannulation, and intramural contrast injection, have been well identified, no validated animal model is available for the study of post-ERCP pancreatitis. In the present study, we induced acute pancreatitis by ERP using CM, and thus, the study shows the devised model offers a means of studying the clinical relation between contrast administration and post-ERCP pancreatitis. Although we did not analyze the effects of CM in detail, such as, the effect of volume or molecular weight, we believe the model could be used to reliably investigate the effects of therapy or means of preventing post-ERCP pancreatitis. On the other hand, TCA induced pancreatitis in current model resembling the situation with biliary pancreatitis. TCA is a deliquescent yellowish crystalline bile acid involved in the emulsification of fats and it occurs as a sodium salt in the bile of human. The technique of retrograde pancreatic ductal infusion of TCA is very similar to the mechanism of clinical biliary pancreatitis which is obstruction of the papilla by gallstones leads to a reflux of bile into the pancreatic duct system. Therefore, our model may be ideally suited for studies exploring the mechanisms related to biliary pancreatitis, as it allows the investigator to use a clinically relevant model in the development of treatment modality.

This study had several limitations. First, only a small number of pigs were included, and although pancreatitis developed in all six animals, larger-scaled study is required to confirm our preliminary results. Second, we did not evaluate the long-term results of acute pancreatitis, because TCA induces severe acute pancreatitis rapidly, and would probably have resulted in mortalities in the long term. Third, we could not evaluate the effect of acute pancreatitis on other organs. Although histologic and hematologic examinations confirmed acute pancreatitis in all animals, analysis of histologic changes in other organs is required to properly assess acute pancreatitis severity because of the systemic inflammatory nature of the disease. Lastly, small amount of $\mathrm{CM}$ was used in all animals to confirm the pancreatic duct cannulation before noxious agent injection. Although the amount of $\mathrm{CM}$ was very small, there is a risk that CM will affect the outcomes in the degree of pancreatitis inducted by TCA.

In conclusion, we developed a large animal model of acute pancreatitis based on the endoscopic administration of CM or TCA into the pancreatic duct. We found the severity of acute pancreatitis was dependent upon the nature and concentration of the agent injected, which indicates that the method might be used to produce pancreatitis of different severities in a controlled manner. We suggest the devised endoscopic technique provides a reliable animal model for those seeking to develop curative and prophylactic treatments for acute pancreatitis.

\section{KEY MESSAGE}

1. Endoscopic retrograde noxious agent injection is effective and safe for creating a model of acute pancreatitis.

2. The severity of acute pancreatitis is depended on the nature of the noxious agent and concentration.

3. These pancreatitis models would be effective for the development of a treatment strategy for acute pancreatitis.

\section{Conflict of interest}

No potential conflict of interest relevant to this article was reported.

\section{Acknowledgments}

The work was supported by Inha University Hospital Research Grant, and the all endoscopic procedures were performed in animal center of National Center of Efficacy Evaluation for the Development of Health Products Targeting Digestive Disorders (NCEED). Seok 
Jeong and Don Haeng Lee both contributed equally to this work and share corresponding authorship.

\section{REFERENCES}

1. Runzi M, Layer P. Etiology, pathogenesis and pathophysiology of acute pancreatitis. Schweiz Med Wochenschr 1997;127:849-853.

2. Chiang DT, Anozie A, Fleming WR, Kiroff GK. Comparative study on acute pancreatitis management. ANZ J Surg 2004;74:218-221.

3. Lerch MM, Gorelick FS. Models of acute and chronic pancreatitis. Gastroenterology 2013;144:1180-1193.

4. Schneider A, Whitcomb DC, Singer MV. Animal models in alcoholic pancreatitis: what can we learn? Pancreatology 2002;2:189-203.

5. Poxleitner PJ, Seifert G, Richter SC, Hopt UT, Wittel UA. Infected pancreatic necrosis increases the severity of experimental necrotizing pancreatitis in mice. Pancreas 2013;42:1150-1156.

6. Hyun JJ, Lee HS. Experimental models of pancreatitis. Clin Endosc 2014;47:212-216.

7. Rongione AJ, Kusske AM, Kwan K, Ashley SW, Reber HA, McFadden DW. Interleukin 10 reduces the severity of acute pancreatitis in rats. Gastroenterology 1997;112:960-967.

8. Ni HB, Ke L, Sun JK, et al. Beneficial effect of hypertonic saline resuscitation in a porcine model of severe acute pancreatitis. Pancreas 2012;41:310-316.

9. Ruben DS, Scorpio DG, Gabrielson KL, Simon BW, Buscaglia JM. Refinement of canine pancreatitis model: inducing pancreatitis by using endoscopic retrograde cholangiopancreatography. Comp Med 2009;59:78-82.

10. Ferrer J, Scott WE 3rd, Weegman BP, et al. Pig pancreas anatomy: implications for pancreas procurement, preservation, and islet isolation. Transplantation 2008;86:15031510.

11. Xie B, Li YY, Jia L, Nie YQ, Du H, Jiang SM. Experimental ablation of the pancreas with high intensity focused ultrasound (HIFU) in a porcine model. Int J Med Sci 2010;8:9-15.

12. Ke L, Ni HB, Tong ZH, Li WQ, Li N, Li JS. Efficacy of continuous regional arterial infusion with low-molecular-weight heparin for severe acute pancreatitis in a porcine model. Shock 2014;41:443-448.

13. Lichtenstein A, Milani R Jr, Fernezlian SM, Leme AS, Capelozzi VL, Martins MA. Acute lung injury in two experimental models of acute pancreatitis: infusion of saline or sodium taurocholate into the pancreatic duct. Crit Care Med 2000;28:1497-1502.

14. Kusske AM, Rongione AJ, Reber HA. Cytokines and acute pancreatitis. Gastroenterology 1996;110:639-642.

15. Donnellan F, Byrne MF. Prevention of post-ERCP pancreatitis. Gastroenterol Res Pract 2012;2012:796751.

16. Aho HJ, Nevalainen TJ, Lindberg RL, Aho AJ. Experimental pancreatitis in the rat. The role of phospholipase A in sodium taurocholate-induced acute haemorrhagic pancreatitis. Scand J Gastroenterol 1980;15:1027-1031.

17. Wittel UA, Wiech T, Chakraborty S, et al. Taurocholate-induced pancreatitis: a model of severe necrotizing pancreatitis in mice. Pancreas 2008;36:e9-e21.

18. Freeman ML. Pancreatic stents for prevention of post-ERCP pancreatitis: the evidence is irrefutable. J Gastroenterol 2014;49:369-370.

19. Marks JM, Dunkin BJ, Shillingstad BL, et al. Pretreatment with allopurinol diminishes pancreatography-induced pancreatitis in a canine model. Gastrointest Endosc 1998;48:180-183. 